\title{
New Suggestions for the Mechanical Control of Bone Remodeling
}

\author{
J. W. C. Dunlop · M. A. Hartmann • \\ Y. J. Bréchet $\cdot$ P. Fratzl $\cdot$ R. Weinkamer
}

Received: 20 October 2008/Accepted: 8 March 2009/Published online: 17 April 2009

(c) The Author(s) 2009. This article is published with open access at Springerlink.com

\begin{abstract}
Bone is constantly renewed over our lifetime through the process of bone (re)modeling. This process is important for bone to allow it to adapt to its mechanical environment and to repair damage from everyday life. Adaptation is thought to occur through the mechanosensitive response controlling the bone-forming and -resorbing cells. This report shows a way to extract quantitative information about the way remodeling is controlled using computer simulations. Bone resorption and deposition are described as two separate stochastic processes, during which a discrete bone packet is removed or deposited from the bone surface. The responses of the bone-forming and -resorbing cells to local mechanical stimuli are described by phenomenological remodeling rules. Our strategy was to test different remodeling rules and to evaluate the time evolution of the trabecular architecture in comparison to what is known from $\mu$-CT measurements of real bone. In particular, we tested the reaction of virtual bone to standard therapeutic strategies for the prevention of bone deterioration, i.e., physical activity and
\end{abstract}

J. W. C. Dunlop and M. A. Hartmann contributed equally to this research.

J. W. C. Dunlop · M. A. Hartmann · P. Fratzl ·

R. Weinkamer $(\square)$

Department of Biomaterials, Max Planck Institute of Colloids and Interfaces, Research Campus Golm, 14424 Potsdam,

Germany

e-mail: richard.weinkamer@mpikg.mpg.de

Present Address:

M. A. Hartmann

Institute of Physics, University of Leoben,

Franz-Josef-Strasse 18, A-8700 Leoben, Austria

Y. J. Bréchet

Groupe Physique du Métal, SIMAP/INPG, Domaine

Universitaire de Grenoble, 38402 Saint Martin d'Hères, France medications to reduce bone resorption. Insensitivity of the bone volume fraction to reductions in bone resorption was observed in the simulations only for a remodeling rule including an activation barrier for the mechanical stimulus above which bone deposition is switched on. This is in disagreement with the commonly used rules having a so-called lazy zone.

Keywords Bone architecture/structure - Bone turnover . Remodeling $\cdot$ Mechanotransduction $\cdot$ Mechanical loading . Exercise $\cdot$ Age $\cdot$ Aging

The strength and fragility of bone are determined by its structure at all levels [1-3], which can be affected by aging, disease, or mechanical damage [4]. As a living organ, bone undergoes a constant renewal process, which helps to maintain its mechanical performance [4] and allows for adaptation to changes in mechanical requirements $[3,5,6]$. Different types of cells are involved in this remodeling process: osteoclasts, which resorb bone, and osteoblasts, which deposit bone [7]. It is generally accepted that bone remodeling is controlled by a mechanosensory system $[8,9]$, and it is considered an interesting example of a homeostatic system where mechanical loads from the outside world control to some extent the amount and the architecture of the bone present in the body.

To describe this, Frost [8] proposed the action of a "mechanostat," which should work in analogy to a thermostat. According to this picture, mechanical disuse would lead to a reduction of the bone mass below a certain set point and loading above a second higher set point, would lead to an increase in bone mass. In between the two set points, sometimes called the "dead" or "lazy zone," the normal healthy value of the bone mass should be maintained [10]. 
This suggests the idea that bone mass may be regulated by constantly monitoring the deviations of the current value of the system from fixed set points [11]. Other interpretations [12], focusing on bone architecture and based on old ideas by Wolff [13] and in particular Roux [14], assume that bone homeostasis is due to an internal regulation network that provides the stability and robustness necessary to react to external stresses. In this picture the equilibrium bone mass is determined by the amount of external load as well as the action of osteoblasts and osteoclasts, which are, in addition to the local loading, regulated by hormones and local biological factors $[8,15,16]$.

Roux's [14] idea of a local mechanical control can be quantitatively defined by phenomenological remodeling rules, which specify the resorption and deposition probabilities as functions of the mechanical stimulus. This results in a local balance without the need of external control by homeostatic set points, in a way somewhat reminiscent of the definition of prices as a balance between offer and demand in a market economy. Since bone resorption is often followed by new bone formation in the same site, the concept of a bone multicellular unit [7, 17] has been proposed. This coordinated replacement of bone is known to occur with a considerable time delay between the resorption, which takes place in weeks, and the formation of new bone, which takes months [7]. While the bone multicellular unit is an attractive picture for bone renewal, it is not sufficient to explain the adaptation of the trabecular bone structure to a changed loading condition. Indeed, adaptation necessarily requires bone to be formed in sites different from those where resorption takes place; otherwise, there would be no modification of architecture. This process, usually referred to as "modeling" [18], can be carried out also in a controlled way since coupling by mechanical strains (which extend over large distances) is in essence nonlocal, so a resorption event in one site and a formation event in a distant site can still influence each other.

The fact that resorption and new bone formation are somewhat dephased, both in time (as they occur at different times in one location) and in space (as they may occur at the same time in different sites during structural adaptation), suggests that stochastic aspects will play a role in the cross-talk between the two processes and will consequently influence the architecture. Indeed, external loads may change in an erratic way during the time of one remodeling cycle, or there might be local variations occurring in biochemical or mechanical signals subjected to noise. Hence, the deterministic view where a sequence of events occurs in a completely predictable fashion must be replaced by probabilities of bone resorption and formation, which will depend on mechanical and biochemical factors. While this seems to be just a technical remark, it has to be emphasized that the stochastic nature of the remodeling rule may have profound effects on bone remodeling and homeostasis as a whole. This is illustrated in Fig. 1 by a hypothetical remodeling event in trabecular bone. A resorption cavity has been induced in the trabecula shown in the right of this figure. This trabecula is now feeling a load higher than average, and according to the mechanism associated with the "mechanostat," bone formation has to take place. This means that-in a completely deterministic picture-path a in Fig. 1 would always be taken, leading to a regenerated trabecula [19]. In a stochastic picture, path a would only be taken with a certain probability and it is not excluded that
Fig. 1 Sketch of a boneresorption event in a trabecula of trabecular bone (left) followed either by new bone formation (path a) or by further resorption (path b) leading to perforation and loss of the trabecula

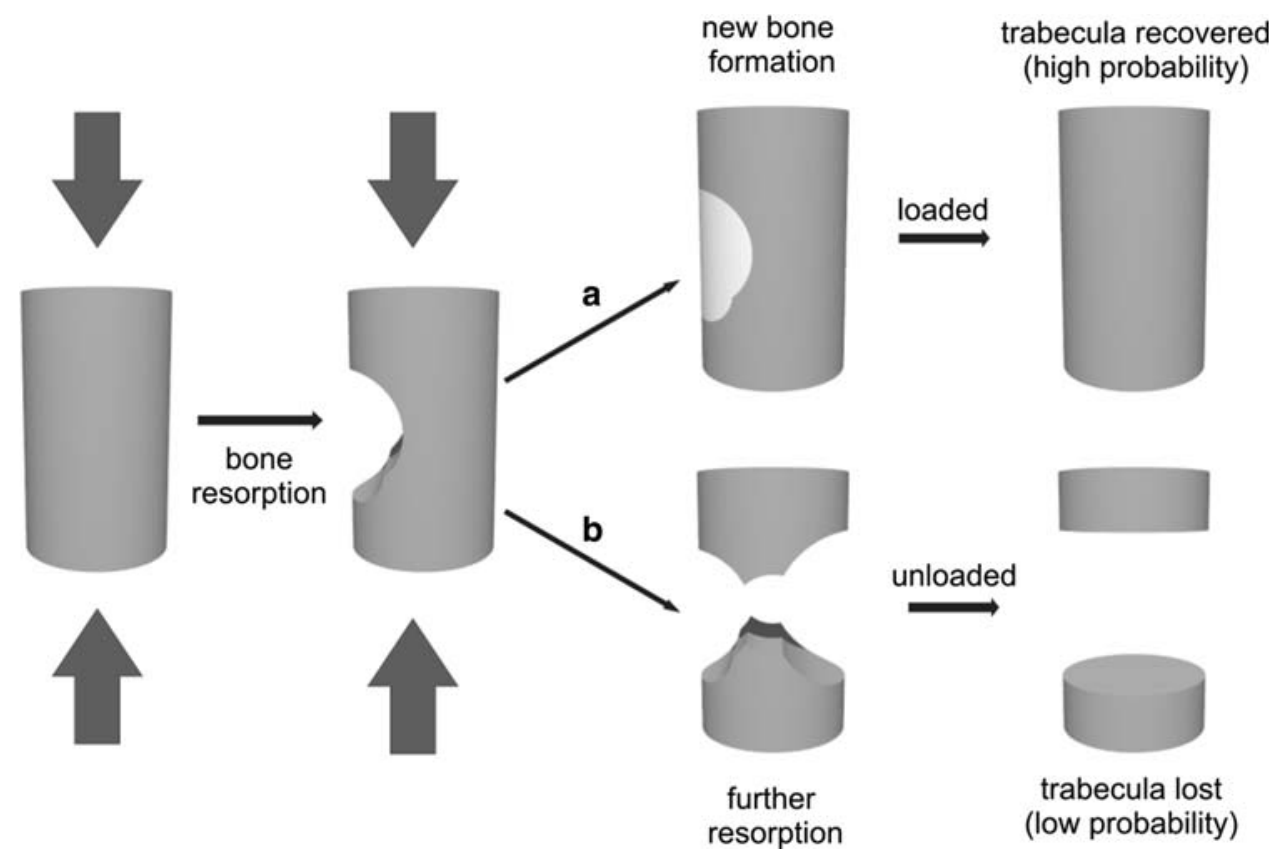


path $b$ could be followed (though with a low probability). Further resorption along path $b$ could lead to a loss of connectivity in this site. This in turn leads to disappearance of the load and of the stimulus for new bone formation in this particular site, making the loss of connectivity irreversible. The probability for path $b$ is significantly increased by the fact that bone remodeling occurs by resorption and deposition of discrete bone packets [20]. Although still improbable, the huge number of remodeling events occurring in our bodies over the years will lead to a modification of trabecular bone architecture, which would not be seen if remodeling was purely deterministic.

It is only recently that experimental methods such as in vivo $\mu$-CT have allowed the observation of time-resolved architectural changes of trabecular bone [21]. However, these experiments are limited to small animals like rats. On the other hand, computer simulations allow us to follow architectural evolution over long periods under controlled conditions. Nevertheless, most computational studies have paid little attention to stochastic effects in combination with the quantized nature of remodeling of bone packets [11, 22-27], with only few exceptions [28-30]. In the present report, we used a computer simulation approach based on a probabilistic description of the bone-remodeling process in terms of resorption and formation probability functions, i.e., remodeling rules, and show that the stochastic nature has profound effects on the time evolution of the trabecular bone structure. In particular, the simulation predicts that the trabecular architecture has a tendency to coarsen with age (by a process not far from what is sketched in Fig. 1), leading to fewer and thicker trabeculae, even when the bone mass stays constant. Although the remodeling rule with a lazy zone was originally proposed by Frost [8] for the control of global bone mass as a function of external load, it has since been used unquestioned to describe local cell behavior. With our model we tested different remodeling rules, including the Frost rule. Comparison of the different model outcomes with morphometric $\mu$-CT data did not allow discrimination of the different rules. The response of virtual bone to changes in loading and bone resorption, however, was different for different remodeling rules. While the Frost rule was not in agreement with experimental data, a remodeling rule comprising an activation barrier displayed the response known from experiment.

\section{Simulation of Bone Remodeling}

The biology of bone remodeling involves numerous biochemical pathways and factors that influence the processes of bone resorption and deposition $[6,15,16]$. Apart from the problem that many details of the mechanoreception, mechanotransduction, and signaling processes are still unknown, the detailed simulation of the complete biochemistry would make simulations of bone remodeling computationally intractable. For the purpose of our simulation, the decision about the resorption or deposition of a bone packet is therefore obtained in three main steps: (1) calculation of the local strains in the bone architecture as a function of external load, (2) estimation of the signal which reaches osteoclasts and osteoblasts, and (3) calculation of the response of these cells in terms of probabilities of bone resorption and deposition.

The forces that a given bone experiences during daily life are difficult to calculate due to the complex geometry of the bone structures themselves as well as the way load is transferred from tendons, muscles, and other bones. As a consequence, the applied external loading is usually assumed to be simple $[22,27,31]$. Nevertheless, calculation of the local strains everywhere in the trabecular architecture is, even under the assumption of isotropic linear elasticity for the bone material, a very computer time-intensive task $[32,33]$. The heterogeneous and hierarchical nature of the bone material, comprising different mineral contents, fiber orientations, and anisotropy of each individual bone packet $[1-3,34]$, means that the actual local strains can only be estimated. Even worse, the simulation of remodeling of trabecular bone inside a human vertebra over the time scale of a human life requires the consideration of approximately 45 million remodeling events and, therefore, at least a similar number of mechanical assessments. Rather than using a full finite element method, we use a fast simplified algorithm to estimate the local strains [31, 35].

The outcome of the second step is the connection between local strains in the bone and the stimulus for bone resorption and deposition at the bone surface. The details of to what mechanical stimulus bone cells react and the underlying mechanism controlling this remain largely unclear. Most probably osteocytes, embedded in the bone matrix and connected by cell processes to other osteocytes and the lining cells at the bone surface [36], take the role of the mechanoreceptive cells responding to fluid flow [37] and/or microdamage $[6,38]$. In vivo experiments demonstrated that changes in applied loading are necessary to induce changes in bone structure [39]. We follow the line of Huiskes [12], who showed that also simulations under static loading can be reinterpreted as the result of bone evolution under dynamic loading. Usually, in simulations the local strains are converted into a scalar quantity, which is referred to as a "mechanical stimulus." From experimental observations it remains open which is the most realistic description for the signaling from the osteocytes inside bone to the bone cells at the surface. Possibilities to model the signal reaching a site at the bone surface include 
summing up all the signals from the immediate surroundings (as chosen in our approach) or considering only the largest signal in the neighborhood. In our model we take the volume change in each neighboring element to be the mechanical signal.

To complete the model, a remodeling rule is needed, which makes the link between the signal that the osteoclasts and osteoblasts actually sense and their response in terms of bone resorption and deposition, respectively. About these reactions no experimental data are yet available. We therefore use the approach of applying different phenomenological remodeling rules to study their effect on the architectural evolution of trabecular bone within a vertebra. Typically, in the literature, resorbed or deposited bone volume is described by a single remodeling rule, which corresponds to the net action of osteoclasts removing and osteoblasts adding bone (e.g., the rule proposed by Frost [10]). It will be demonstrated that a definition of two separate remodeling rules for bone resorption and deposition is crucial for trabecular bone. In the present report, the remodeling rules are defined as a function of mechanical stimulus only, but an extension to include biological stimuli (e.g., hormones, drugs) is straightforward. As the model is stochastic, the remodeling rules that were used (sketched in Fig. 2a-d and described in Table 1) define only the probability of resorption or deposition of bone at its surface.
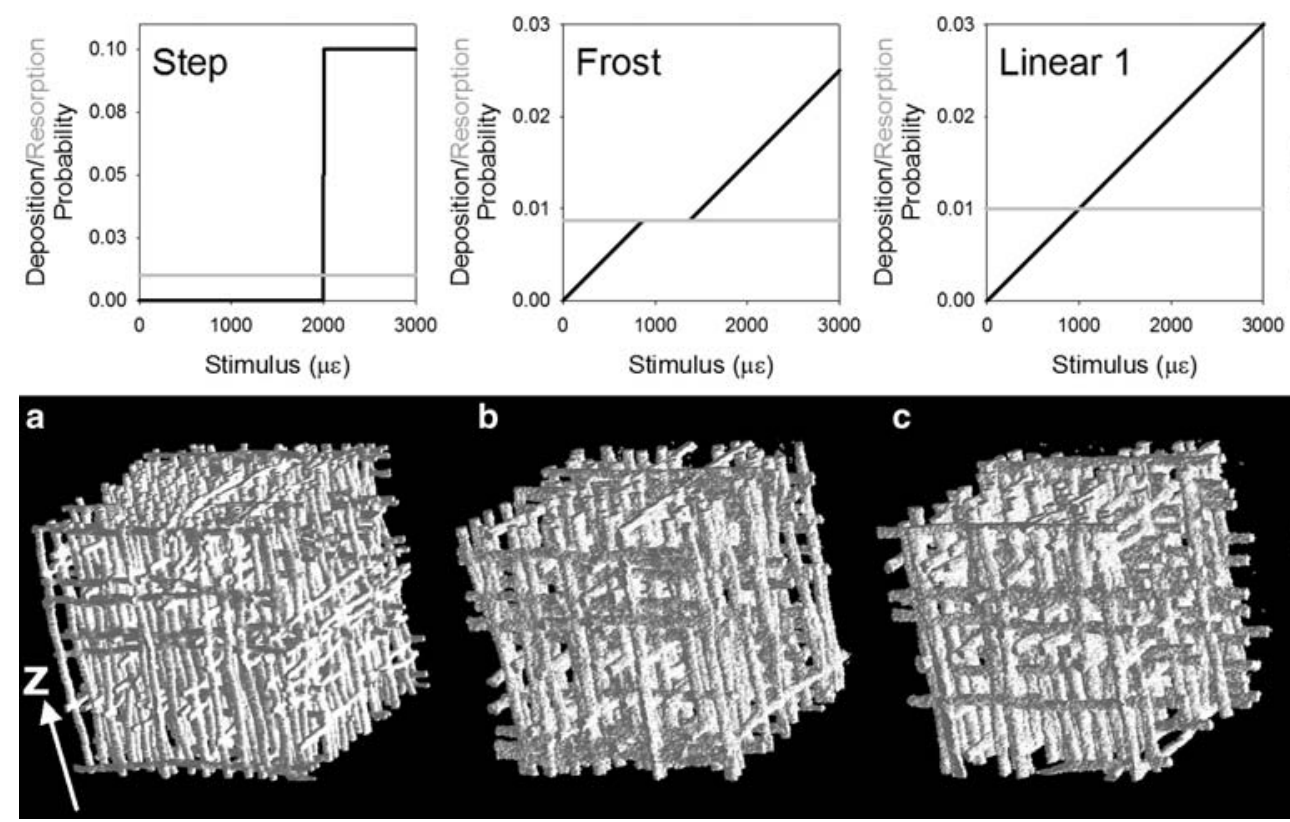

Fig. 2 a-d Bone deposition (black) and resorption (gray) probabilities for the different remodeling rules investigated (for numerical values, see also Table 1). Upper images illustrate the remodeling rules used: a step, b Frost, $\mathbf{c}$ linear 1, and $\mathbf{d}$ linear 2. Remodeling rules $\mathbf{a}-\mathbf{c}$ all use constant bone resorption probabilities. Remodeling rule $\mathbf{d}$ has a linear response for both deposition and resorption resulting in a

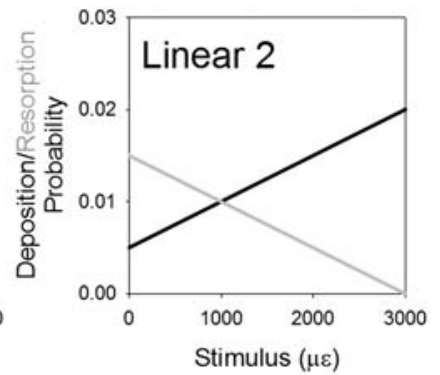

\section{Methods}

Simulations incorporating different remodeling rules were run using a random starting configuration, at an initial bone volume fraction of $95 \%$. Four different remodeling rules (step, Frost, linear 1 and 2; see Fig. 2 and Table 1 for numerical values) were tested in a simple cubic lattice with 256 voxels in each dimension. Occupied sites on the lattice correspond to sites filled with bone and unoccupied sites, to ones filled with marrow. Since the trabecular bone inside a human vertebra should be modeled, the system was surrounded by a layer of (cortical) bone, which was not allowed to remodel. The box was loaded homogeneously in the vertical direction. Due to the inwaisting form of a vertebral body, which makes the sides of the vertebra bulge inward, it was assumed that the vertical load causes also effective loads in the two horizontal directions (for explicit expressions, see [31]), resulting in an effective triaxial loading with its main force in the vertical direction. The local strains were estimated using first a two-way painting algorithm, which simulates the transmission of the force through the network-like architecture to determine the loaded elements in the trabecular structure [31, 35]. The idea is inspired by the field of porous or granular media in which forces are seen to "flow" like electrical currents through structures [40]. The local strain was then calculated under the assumption that the resulting strain in a

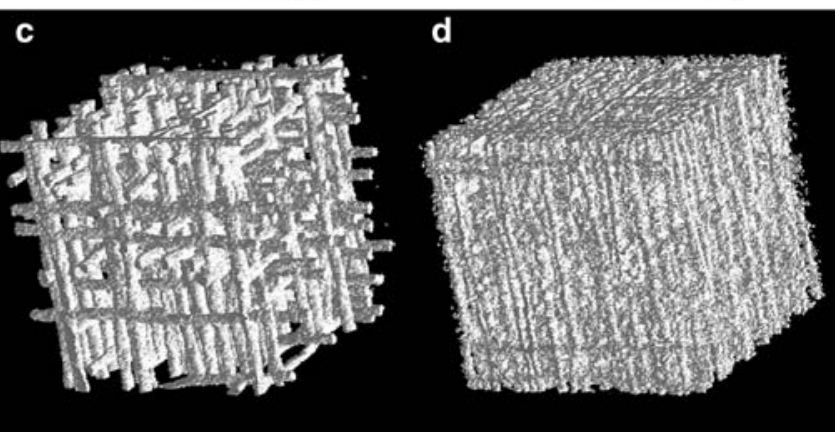

net response which is equivalent to c. Lower images show the simulation output after 40 years for the different remodeling rules (ad) applied on a cubic lattice of dimensions $256 \times 256 \times 256$ and a voxel size of $17 \mu \mathrm{m}$. Arrow marks the main loading direction along the spine 
Table 1 Equations for the four different remodeling rules and the parameters used in the simulations

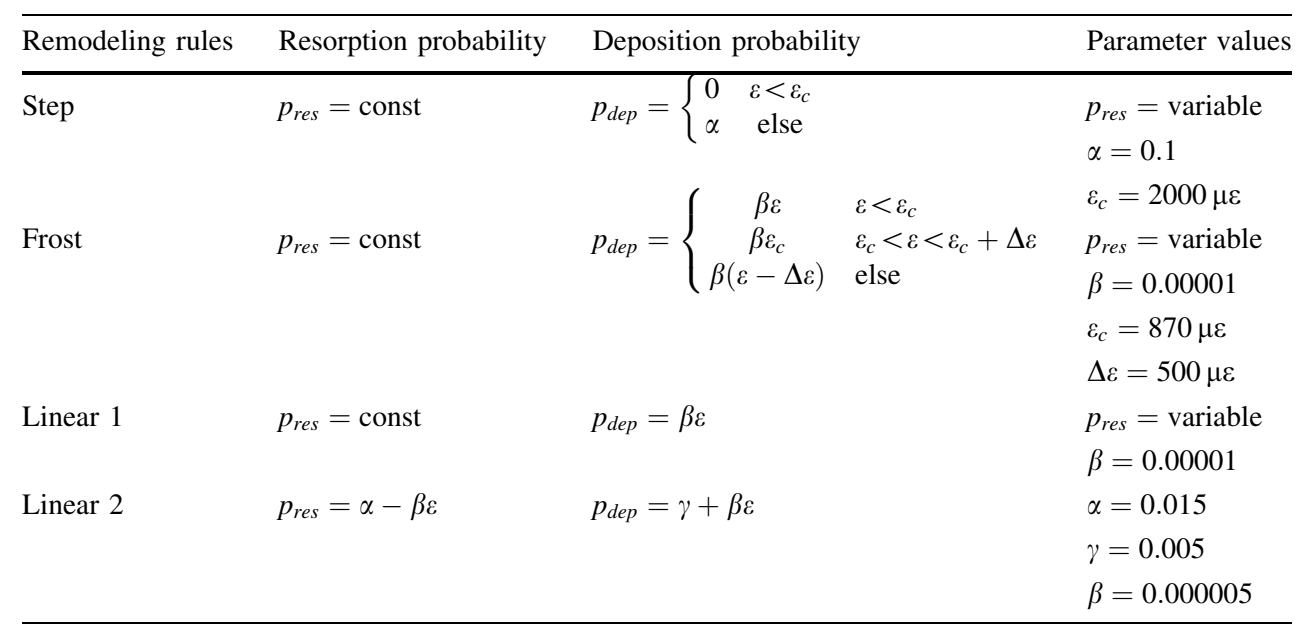

The different remodeling rules are described by two equations, one giving the probability of resorption $\left(p_{\text {res }}\right)$ and the other giving the probability for deposition $\left(p_{d e p}\right)$ as a function of local strain be homogeneous and isotropic with a Young's modulus of $15 \mathrm{GPa}[5]$.

tional area. This procedure was repeated in all three loading directions, yielding as a result the total volume change in each cubic bone element of the structure. Although the mechanics are not as accurate as the finite element method, the algorithm is about two orders of magnitude faster, enabling simulations to run on large system sizes for many time steps and for detailed parametric studies to be done on the effect of different remodeling hypotheses. The individual remodeling event was simulated by first choosing a site at the bone-marrow interface at random. The mechanical stimulus for the resorption/deposition event was obtained by summing up the total volume change in the nearest neighbor voxels of the chosen lattice site. With the mechanical stimulus as input, the remodeling rule provides the probability for a remodeling event (see Table 1 for remodeling rules used). A random number, equally distributed in the interval between 0 and 1 was drawn. If this number was smaller than the deposition/ resorption probability, the state of the investigated element was changed (occupied to unoccupied in the case of resorption or vice versa in the case of deposition). The free parameters in the definition of the remodeling rules were chosen in such a way that the steady-state value of the bone volume fraction is the same for each of the simulations. The conversion of computer time to real time was performed knowing that the turnover time, i.e., the time necessary to remodel a bone volume equal to the present volume, is about 4 years in human vertebrae [7]. The mean trabecular thickness after 30 years was assumed to be $125 \mu \mathrm{m}$ [41], which corresponds to a voxel side length of $17 \mu \mathrm{m}$ and a total system side length of $0.5 \mathrm{~cm}$ of the virtual bone. The applied stress on the system was chosen to be the same as that on a vertebra during physical activity, about 2,000 $\mathrm{N}$ [42] on an area of $\sim 1,200 \mathrm{~mm}^{2}$, i.e., a stress of 1.7 MPa. The bone material was assumed to

\section{Results}

We report on the simulation of architectural changes within a human vertebra during a lifetime obtained by incorporating different remodeling rules (Fig. $2 \mathrm{a}-\mathrm{d}$, Table 1). Simulations were run using a randomized starting configuration, at an initial bone volume fraction of $95 \%$. All simulations result in the emergence of a network-like structure consisting of rod-shaped trabeculae. The trabeculae are preferentially oriented along the main loading directions along the spine and perpendicular to it, given rise to a strong architectural anisotropy (Fig. 2, bottom). Although all simulated architectures after 40 years of remodeling have the same bone volume fraction, the architecture is very different for different remodeling rules. The step remodeling rule (Fig. 2a) forms many more smoother and smaller trabeculae than both the linear (Fig. 2c) and Frost (Fig. 2b) rules. The Frost rule leads to the development of a much rougher surface compared to both the linear and step rules. Figure $2 d$ shows an image after 40 years of a simulation that has been run with the same linear net remodeling rule as Fig. 2c but made up with different resorption and deposition responses. A much finer structure is observed, with three times as many trabeculae but with one-third the thickness of those seen in Fig. 2c. The bone volume fraction (BV/TV) is more than a factor 2 higher (see also right part of Fig. 3).

More quantitative data from the dynamic behavior during simulations is given in Fig. 3. BV/TV drops rapidly from the initially high value of the starting configuration, tending toward a steady-state value of around $15 \%$. The independence of this steady-state value of BV/TV from the initial 
Fig. 3 Time evolution of morphological parameters for the simulations considered in Fig. 2a-c (step, Frost, and linear 1) on the left and Fig. $2 \mathrm{~d}-\mathrm{f}$ (linear 1 and linear 2) on the right. $B V / T V$ trabecular bone volume fraction, $T b . N$ average number of trabeculae per millimeter in the principal loading direction, Tb.Th average trabecular thickness. Error bars give the standard deviation from three independent simulations
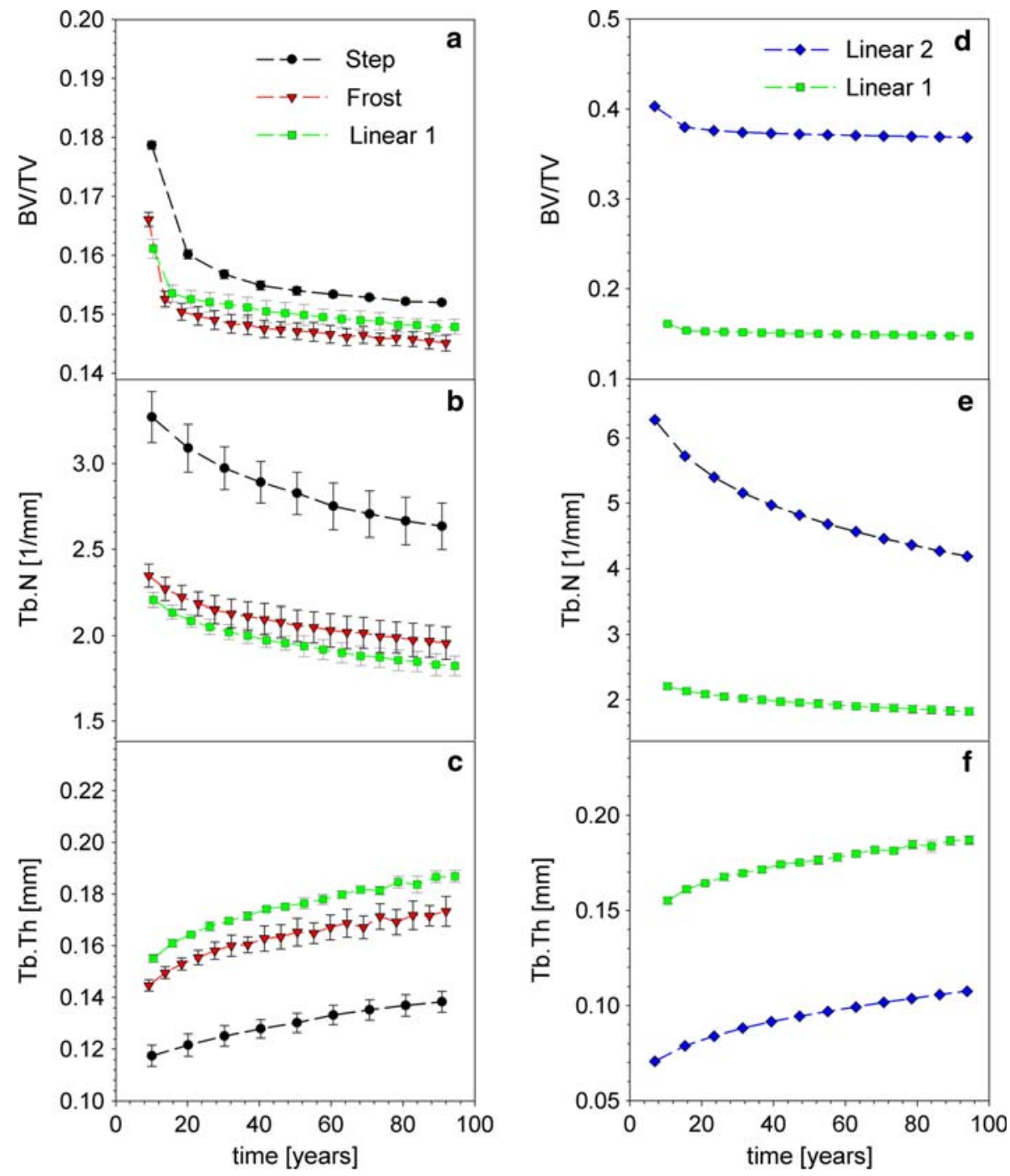

condition was tested by starting simulations with a BV/TV below the steady-state value. Concerning bone mass, the virtual bone therefore reaches homeostasis. However, the system is in dynamic structural equilibrium as reflected in standard bone morphogenetic quantities. The trabecular number decreases with time, while mean trabecular thickness increases (Fig. 3b, c). The rate of decrease of trabecular number beyond 40 years is $4.3 \times 10^{-3} \mathrm{~mm}^{-1}$ year ${ }^{-1}$ for the step and $2.6 \times 10^{-3} \mathrm{~mm}^{-1}$ year ${ }^{-1}$ for both the Frost and the linear remodeling rules. Typical coarsening velocities (trabecular thickness increase per year) are $1.8 \times$ $10^{-4} \mathrm{~mm} \mathrm{year}^{-1}$ (step), $2.1 \times 10^{-4} \mathrm{~mm} \mathrm{year}^{-1}$ (Frost), and $2.4 \times 10^{-4} \mathrm{~mm} \mathrm{year}^{-1}$ (linear) (see also Table 2).

The hypothesis that a local mechanical control of the remodeling process should be reflected in the variation of structural features was tested by analyzing the distribution of the trabecular cross-sectional areas (Fig. 4). With time the distributions shift in position toward larger cross sections (Fig. 4a), implying that not only are small trabeculae
Table 2 Coarsening rates for the decrease in $\mathrm{Tb} . \mathrm{N}$ and the increase in $\mathrm{Tb} . \mathrm{Th}$

\begin{tabular}{lll}
\hline Remodeling rules & $\begin{array}{l}\text { Rate of decrease } \\
\text { of Tb.N }\left(\mathrm{mm}^{-1} \text { year }^{-1}\right)\end{array}$ & $\begin{array}{l}\text { Rate of increase } \\
\text { of Tb.Th }\left(\mathrm{mm} \mathrm{year}^{-1}\right)\end{array}$ \\
\hline Step & $4.3 \times 10^{-3}$ & $1.8 \times 10^{-4}$ \\
Frost & $2.6 \times 10^{-3}$ & $2.1 \times 10^{-4}$ \\
Linear 1 & $2.6 \times 10^{-3}$ & $2.4 \times 10^{-4}$ \\
Stauber-Müller & $4.8 \times 10^{-3}$ & $1.2 \times 10^{-4}$ \\
\hline
\end{tabular}

Shown are the rates obtained for the three investigated remodeling rules as well as the experimental results extracted from the data in [41]

being lost but the remaining trabeculae become thicker. While this coarsening of the structure occurs, the shape of the distribution practically remains unchanged. Independent of the remodeling rule, the trabecular area distributions display a similar bell-shaped form (Fig. 4b). For the step remodeling rule, the peak in distribution is at smaller 
Fig. 4 a Evolution of trabecular area (Tb.A) distributions for the step remodeling rule. b Comparison of trabecular area distributions for the different remodeling rules after 30 simulated years of bone remodeling. Gray bars denote the mean of seven trabecular area distributions of healthy lumbar vertebrae measured by $\mu$-CT with $14 \mu \mathrm{m}$ resolution (courtesy of Müller et al.)

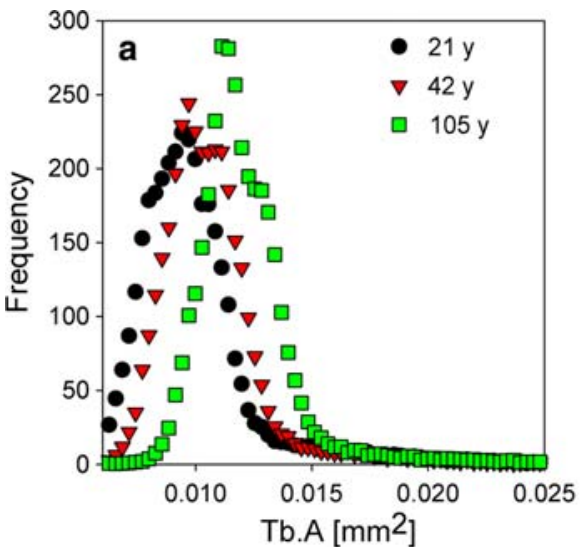

values for the trabecular area and is much narrower than for the other remodeling rules.

Of great practical importance is how the mechanosensitive system in bone reacts to changes in mechanical loading (corresponding to physical exercise [43]) and to reduction in osteoclast resorption probability (corresponding to therapy with antiresorptive agents [44]). The effect on the resultant morphometric parameters for each of the remodeling rules is plotted in Fig. 5. An increasing external load due to physical exercise causes a linear increase in equilibrium BV/TV for all simulations (Fig. 5a). This linear increase is identical for all remodeling rules so that a

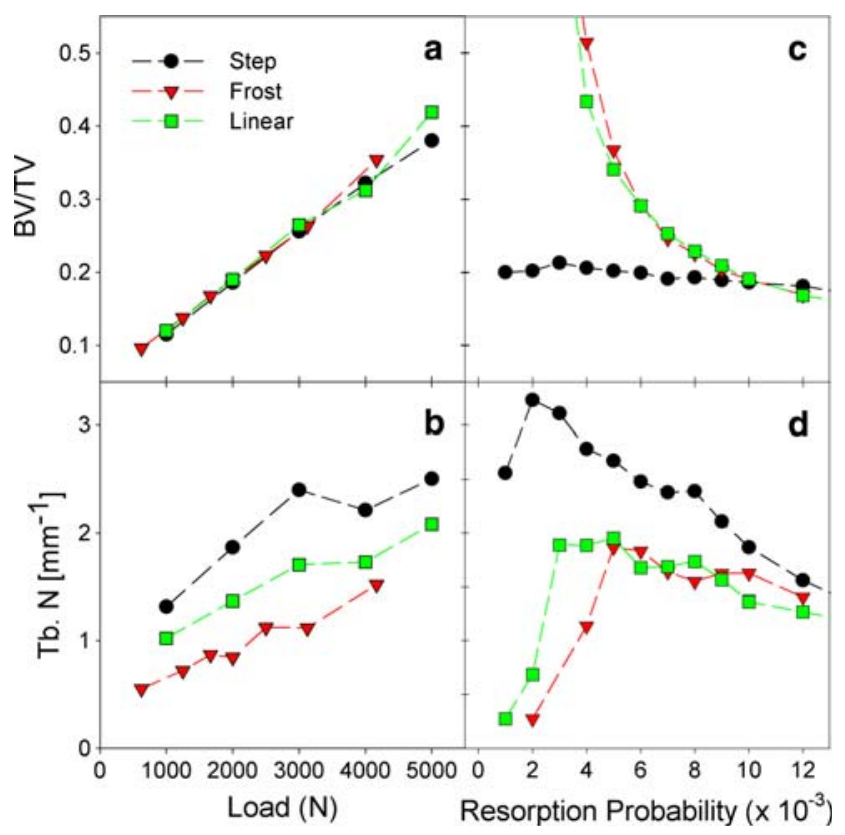

Fig. 5 a-d Sensitivity of morphological parameters (steady-state BV/TV and Tb.N after 40 years) to changing external load with constant resorption probability $(=0.01)(\mathbf{a}, \mathbf{b})$ and changing resorption probability for constant load $(=2,000 \mathrm{~N})(\mathbf{c}, \mathbf{d})$, for the three different remodeling rules (step, Frost, and linear 1). The decrease in trabecular number at low resorption probabilities in (d) is due to a very high value for $\mathrm{BV} / \mathrm{TV}$ (c)
$10 \%$ increase in mechanical load results in an increase of about $8 \%$ in BV/TV. The response to a reduction in bone resorption probability by osteoclasts, $\mathrm{p}_{\text {res }}$, however, is significantly different for different remodeling rules. The step remodeling rule is almost insensitive to the decrease of the resorption probability, whereas both the linear and Frost remodeling rules show a monotonic increase in BV/ TV with decreasing bone resorption (Fig. 5c). Further simulations using the step remodeling rule show that this insensitive response of $\mathrm{BV} / \mathrm{TV}$ to changes in $\mathrm{p}_{\text {res }}$ is generic to this rule. The trabecular number is consistently higher for the step remodeling rule than the two others regardless of the applied load and resorption probability (Fig. 5b, d).

\section{Discussion}

Remodeling of trabecular bone can be characterized as (1) a mechanically controlled process, which is (2) stochastic and (3) quantized in nature leading to the resorption/ deposition of a discrete bone packet during a remodeling cycle [20]. These three characteristics have been implemented in a computer model where mechanical control of the process occurs due to the local mechanics described by remodeling rules. The computational results showed that bone volume converges toward a steady-state value. In contrast to deterministic simulations, where also the architecture attains a homeostatic configuration in the sense of an optimized topology, the stochastic and quantum nature of our model results in a constant loss of trabeculae. This coarsening of the trabecular architecture with a constant loss of trabeculae due to events such as those illustrated in Fig. 1 can be interpreted as a natural result of aging.

Recent morphological analysis of vertebral trabecular bone using $\mu$-CT showed that with age trabecular number (Tb.N) decreases, while trabecular thickness (Tb.Th) increases in a lumbar vertebra (but interestingly not in the femoral head) [41]. Figure 6 compares the development of 
Fig. 6 a-d Comparison of the simulation (solid lines) with experimental values (black dots) for the most important morphological parameters as a function of age. Dashed gray lines give a LOWESS fit of the experimental data. a Normalized BV/TV, b normalized BS/TV, c normalized Tb.N, and $\mathbf{d}$ normalized Tb.Th. Data taken from [41]

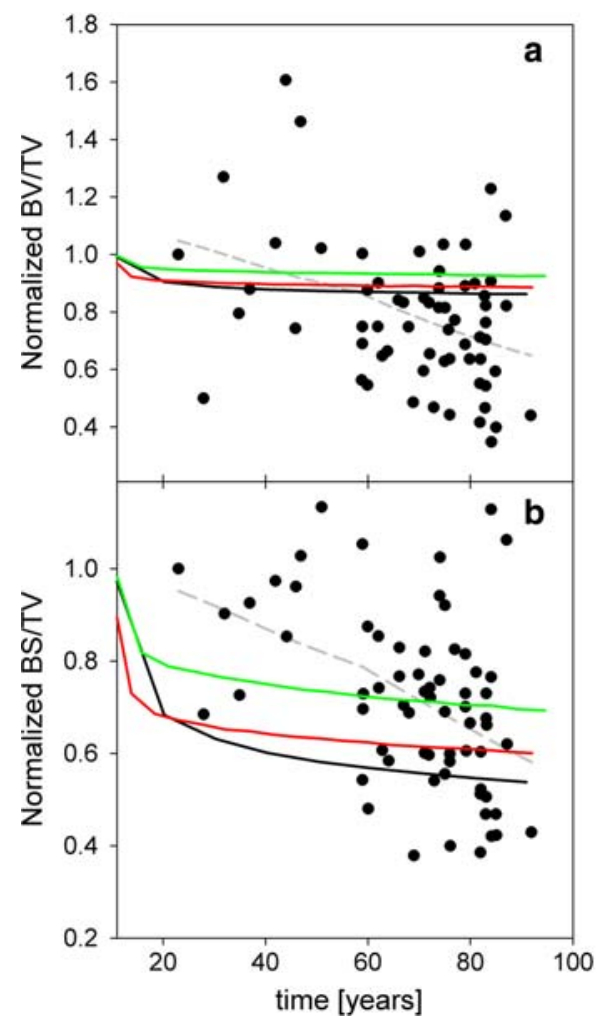

BV/TV, Tb.N, Tb.Th, and BS/TV (bone surface divided by tissue volume) with age obtained by the simulation (solid lines) and measured by $\mu$-CT for the lumbar spine (black dots) (as given in [41]). The measured data shown are the combined results for men and women. Both data sets were normalized to the first measured point. Using a linear fit of the original data, the experimentally determined rate of reduction in trabecular number in the vertebra is about $4.8 \times 10^{-3} \mathrm{~mm}^{-1}$ year $^{-1}$; the corresponding experimental value for the coarsening velocity is approximately $1.2 \times 10^{-4} \mathrm{~mm} \mathrm{year}^{-1}$ (see also Table 2). Both values are in reasonable agreement with the simulations, although a comparison does not allow singling out of one remodeling rule that fits the data best. This is also because the scatter of the experimental data in this cross-sectional study is quite significant.

While our simulation predicts coarsening velocities close to the experimentally observed ones, vertebral bone volume fractions tend toward a steady-state BV/TV, while experimentally a decrease with age at a rate of $6.4 \times 10^{-4}$ year $^{-1}$ was observed. In our model this decrease can be explained as a result either of a reduced external load or of a change in the remodeling rules like a decrease in the slope or a shift of the step in the linear or the step-like remodeling rule for bone deposition, respectively, both leading to a reduced bone volume fraction. This observation follows the line of the Utah paradigm of the mechanostat, where a key argument is the possibility of changes of the set points with hormonal changes or administration of medications [45] or a reduced loading due to decreasing muscle strength with age [10] (see also Fig. 5). Our model offers therefore a framework to distinguish between architectural changes due to aging (coarsening for an unchanged remodeling rule) and due to disease (loss of bone volume via changing the remodeling rule).

In Fig. 4b simulated trabecular area distributions are compared to a measured one. We are not aware of any such comparison already presented. The experimental distribution was obtained from the original $\mu$-CT data (presented in [41]). Seven data sets with a resolution of $14 \mu \mathrm{m}$ of persons aged between 20 and 40 years were used to obtain the averaged distribution. The area of all distributions is normalized to one. The measured trabecular area distribution displays a peak at a position close to the position of the peak obtained by the simulation using the step remodeling rule. Nevertheless, the measured distribution is much broader, meaning the peak height is much lower than for the simulated distributions. This discrepancy probably arises due to the existence of plate-like trabeculae, which are found in real bone. Comparing the trabecular area distributions obtained with the three different remodeling rules, it is striking that the distribution for the step remodeling rule is centered at a lower trabecular area and is narrower than for the two other remodeling rules. This observation can be understood by the degree of control 
imposed by the different remodeling rules. An on-off control corresponding to the step remodeling rule is the strictest control and results therefore in a narrow trabecular area distribution.

Further an important result is that the actions of bone resorption and deposition have to be described in two separate control rules instead of lumping them together in one net-remodeling rule. Figs. 2 and 3 (right part) show that strong differences observed in morphogenetic parameters can be attributed not only to different remodeling rules (Fig. 2a-c) but even to rules with the same net response (Fig. 2c, d), which differ only in the subdivision between bone resorption and deposition.

Architectural details of the structural development of vertebral trabecular bone are not reproduced by our model. In the literature the trabecular architecture inside a vertebral body is reported to change with age from a plate-like to a rod-like architecture [41]. In our simulations no plates in the structure were formed. While it is possible that plates result only for specific remodeling rules (that we did not test), the reason for the observation of no plates in the simulations should be looked for in necessary model simplifications. Although the loading of a vertebra is rather simple compared to other bones in the human skeleton, it is surely more complex than assumed in the simulations [42], i.e., a constant triaxial loading with the main loading along the vertical direction of the spine. One further has to keep in mind that bone is "servant of more than one master," fulfilling functions beyond providing mechanical stability. The importance of especially trabecular bone as an easily accessible calcium reservoir has been pointed out [46, 47]. It is likely that the absence of plates in the simulated structure explains also the deviations of the simulated BS/ TV from the experimental one (Fig. 6b). At early times the change in the experimental BS/TV is most probably due to changes in topology, which are not present in the simulations.

While comparison of the simulated and measured time development of morphological parameters does not allow for a distinction of the different remodeling rules, the reaction of the virtual bone to physical exercise and antiresorptive therapy is revealing. In the case of changes in the external load, all the different remodeling rules show the same behavior (Fig. 5a). This is in particular surprising for the Frost remodeling rule (Fig. 2b) as it has a lazy zone in which the combined action of bone resorption and deposition cancels out, resulting in a zero net action. A remodeling rule with such a lazy zone was designed exactly for the purpose of guaranteeing a constant bone volume within the load window defined by the lazy zone [10] and has been frequently used in simulations. Our simulations show that a stochastic remodeling process introduces a natural variability into the system, giving a distribution in the size of trabeculae and, hence, local strains (Fig. 4). This has the consequence that, although the average strain (mechanical stimulus) may lie in the center of the lazy zone, a change in load will cause some trabeculae to have a stimulus outside the bounds of the lazy zone, in turn resulting in net deposition or resorption and a change in bone volume.

A clear distinction between the remodeling rules can be found, however, for the case of antiresorptive therapy. Experimental data of the bisphosphonate risedronate show a reduction of the resorption probability of $50 \%$, while the bone volume fraction does not change significantly [4850]. This behavior is in clear contradiction to simulation results using a linear or Frost remodeling rule but agrees with results obtained with the step remodeling rule. Our simulations therefore provide a clear indication that the mechanosensitive system in bone makes use of an activation barrier to control the remodeling process. In conclusion, our work shows (1) there are several different remodeling rules which lead to the emergence of trabecular-like patterns in the simulation of bone remodeling, (2) the experimentally observed coarsening of bone architecture can be explained by the stochastic nature of the remodeling process and occurs independently of the three tested remodeling rules, and (3) comparison with data for reductions in bone resorption showed that a step-like remodeling results in the best agreement. The exact form of the remodeling rule is of crucial importance to understand and to predict the reaction of bone to medical treatment.

Acknowledgments We gratefully acknowledge Ralph Müller, Martin Stauber, and Davide Ruffoni for supplying the data of the experimental trabecular bone distribution. Technical assistance by Peter Saparin and Carolin Lukas is acknowledged. J. W. C. Dunlop thanks the Alexander von Humboldt Foundation for a postdoctoral fellowship.

Open Access This article is distributed under the terms of the Creative Commons Attribution Noncommercial License which permits any noncommercial use, distribution, and reproduction in any medium, provided the original author(s) and source are credited.

\section{References}

1. Weiner S, Wagner HD (1998) The material bone: structuremechanical function relations. Annu Rev Mater Sci 28:271-298

2. Fratzl P, Gupta HS, Paschalis EP, Roschger P (2004) Structure and mechanical quality of the collagen-mineral nano-composite in bone. J Mater Chem 14:2115-2123

3. Fratzl P, Weinkamer R (2007) Nature's hierarchical materials. Prog Mater Sci 52:1263-1334

4. Taylor D, Hazenberg JG, Lee TC (2007) Living with cracks: damage and repair in living bone. Nat Mater 6:263-268

5. Currey JD (2002) Bones, structure and mechanics. Princeton University Press, Princeton 
6. Robling AG, Castillo AB, Turner $\mathrm{CH}$ (2006) Biomechanical and molecular regulation of bone remodeling. Annu Rev Biomed Eng 8:455-498

7. Eriksen EF (1986) Normal and pathological remodeling of human trabecular bone-3-dimensional reconstruction of the remodeling sequence in normal and in metabolic bone-disease. Endocr Rev 7:379-408

8. Frost HM (1987) Bone mass and the mechanostat-a proposal. Anat Rec 219:1-9

9. Rodan GA (1997) Bone mass homeostasis and bisphosphonate action. Bone 20:1-4

10. Frost $H$ (1997) On our age-related bone loss: insights from a new paradigm. J Bone Miner Res 12:1539-1546

11. Turner $\mathrm{CH}$ (1991) Homeostatic control of bone structure: an application of feedback theory. Bone 12:203-217

12. Huiskes R (2000) If bone is the answer, then what is the question? J Anat 197:145-156

13. Wolff $\mathbf{J}$ (1892) The law of bone remodeling [in German]. Springer, Berlin

14. Roux W (1881) Der Kampf der Theile im Organismus. Wilhelm Engelman, Leipzig

15. Manolagas SC (2000) Birth and death of bone cells: basic regulatory mechanisms and implications for the pathogenesis and treatment of osteoporosis. Endocr Rev 21:115-137

16. Harada S, Rodan GA (2003) Control of osteoblast function and regulation of bone mass. Nature 423:349-355

17. Parfitt AM (1994) Osteonal and hemi-osteonal remodeling-the spatial and temporal framework for signal traffic in adult human bone. J Cell Biochem 55:273-286

18. Frost H (1990) Skeletal structural adaptations to mechanical usage (SATMU): 1. Redefining Wolff's law: the bone modeling problem. Anat Rec 226:403-413

19. Smit TH, Burger EH (2000) Is BMU-coupling a strain-regulated phenomenon? A finite element analysis. J Bone Miner Res 15:301-307

20. Parfitt AM (1984) The cellular basis of bone remodeling: the quantum concept reexamined in light of recent advances in the cell biology of bone. Calcif Tissue Int 36:S37-S45

21. Waarsing JH, Day JS, Van der Linden JC, Ederveen AG, Spanjers C, De Clerck N, Sasov A, Verhaar JAN, Weinans H (2004) Detecting and tracking local changes in the tibiae of individual rats: a novel method to analyse longitudinal in vivo micro-CT data. Bone 34:163-169

22. Huiskes R, Ruimerman R, van Lenthe GH, Janssen JD (2000) Effects of mechanical forces on maintenance and adaptation of form in trabecular bone. Nature 405:704-706

23. van Oers RFM, Ruimerman R, Tanck E, Hilbers P, Huiskes R (2008) A unified theory for osteonal and hemi-osteonal remodeling. Bone 42:250-259

24. Cowin SC (1993) Bone stress-adaptation models. J Biomech EngT ASME 115:528-533

25. Tsubota K-I, Adachi T, Tomita Y (2002) Functional adaptation of cancellous bone in human proximal femur predicted by trabecular surface remodeling simulation toward uniform stress state. J Biomech 35:1541-1551

26. Thomsen J, Mosekilde L, Boyce RW, Mosekilde E (1994) Stochastic simulation of vertebral trabecular bone remodeling. Bone 15:655-666

27. Ruimerman R, Hilbers P, Van Rietbergen B, Huiskes R (2005) A theoretical framework for strain-related trabecular bone maintenance and adaptation. J Biomech 38:931-941

28. Müller R (2005) Long-term prediction of three-dimensional bone architecture in simulations of pre-, peri- and post-menopausal microstructural bone remodeling. Osteoporos Int 16:S25-S35
29. Van der Linden JC, Verhaar JAN, Weinans H (2001) A threedimensional simulation of age-related remodeling in trabecular bone. J Bone Miner Res 16:688-696

30. Tayyar S, Weinhold PS, Butler RA, Woodard JC, Zardiackas LD, St John KR, Bledsoe JM, Gilbert JA (1999) Computer simulation of trabecular remodeling using a simplified structural model. Bone 25:733-739

31. Weinkamer R, Hartmann MA, Brechet Y, Fratzl P (2004) Stochastic lattice model for bone remodeling and aging. Phys Rev Lett 93:228102

32. VanRietbergen B, Weinans H, Huiskes R, Polman BJW (1996) Computational strategies for iterative solutions of large FEM applications employing voxel data. Int J Numer Methods Eng 39:2743-2767

33. Homminga J, Van-Rietbergen B, Lochmüller EM, Weinans $H$, Eckstein F, Huiskes R (2004) The osteoporotic vertebral structure is well adapted to the loads of daily life, but not to infrequent "error" loads. Bone 34:510-516

34. Zysset PK (2003) A review of morphology-elasticity relationships in human trabecular bone: theories and experiments. $\mathrm{J}$ Biomech 36:1469-1485

35. Parkinson J, Brass A, Canova G, Brechet Y (1997) The mechanical properties of simulated collagen fibrils. J Biomech 30:549-554

36. Cowin SC, Moss ML (2001) Mechanosensory mechanisms in bone. In: Cowin SC (ed) Bone mechanics handbook. CRC Press, Boca Raton

37. Burger EH, Klein-Nulend J (1999) Mechanotransduction in bone-role of the lacuno-canalicular network. FASEB J 13:S101-S112

38. Mori S, Burr DB (1993) Increased intracortical remodeling following fatigue damage. Bone 14:103-109

39. Lanyon LE, Rubin CT (1984) Static vs dynamic loads as an influence on bone remodelling. J Biomech 17:897-905

40. Zuriguel I, Mullin T, Rotter JM (2007) Effect of particle shape on the stress dip under a sandpile. Phys Rev Lett 98:028001

41. Stauber M, Müller R (2006) Age-related changes in trabecular bone microstructures: global and local morphometry. Osteoporos Int 17:616-626

42. Adams MA, Dolan P (1995) Recent advances in lumbar spinal mechanics and their clinical significance. Clin Biomech 10:3-19

43. NIH (2000) Osteoporosis prevention, diagnosis, and therapy. NIH consensus statement, vol 17. NIH, Bethesda, pp 1-45

44. Rodan GA, Martin TJ (2000) Therapeutic approaches to bone diseases. Science 289:1508-1514

45. Frost H (1998) Changing concepts in skeletal physiology: Wolff's law, the mechanostat and the "Utah paradigm". Am J Hum Biol 10:599-605

46. Parfitt AM (2003) Misconceptions III. Calcium leaves bone only by resorption and enters only by formation. Bone 33:259-263

47. Parfitt AM (1989) Plasma calcium control at quiescent bone surfaces: a new approach to the homeostatic function of bone lining cells. Bone 10:87-88

48. Fratzl P, Roschger P, Fratzl-Zelman N, Paschalis EP, Phipps R, Klaushofer K (2007) Evidence that treatment with risedronate in women with postmenopausal osteoporosis affects bone mineralization and bone volume. Calcif Tissue Int 81:73-80

49. Perez-Lopez FR (2004) Postmenopausal osteoporosis and alendronate. Maturitas 48:179-192

50. Weinstein RS, Parfitt AM, Marcus R, Greenwald M, Crans G, Muchmore DB (2003) Effects of raloxifene, hormone replacement therapy, and placebo on bone turnover in postmenopausal women. Osteoporos Int 14:814-822 